\title{
RECENSIONES
}

\section{Editiones externae}

Láng Orsolya-Nagy Alexandra-Vámos Péter: The Aquincum Macellum. Researches in the area of the macellum in the Aquincum Civil Town (1882-1965). Applying new methods for old excavation materials. Aquincum Nostrum I. ISSN: 1217-3592, 3. Budapest History Museum. Budapest, 2014. ISBN: 978-615-5341-17-5. 198 pages with 42 figures and 44 tables

Recently has been released within the publication seires of the Budapest History Museum, Aquincum Nostrum I.3, the work of three distinguished reserchers, which is a synthesis about the macellum in the Aquincum Civil Town, based on the old excavations, that took place here between 1882 and 1965 . There were publications, which already discussed the different aspects of this building, but this time the authors worked as a team of specialists to sum up all the information about previous researches and of those finds, which were unpublished untill now.

The authors have considerable experience with materials from this site, as they are archaeologists of the Aquincum Museum. Orsolya Láng is a researcher of the Aquincum Civil Town, and the presented work is mainly based on her MA thesis written in 2001. Péter Vámos is a specialist of local pottery production and generally of roman pottery of Aquincum. Alexandra Nagy is also a pottery specialist, her field of interest covers the range of Pannonian grey wares and stamped pots. It is necessary to mention the contribution of Anna Biller, who wrote chapter number 7.4 about archaeozoological finds.

The topic seems to be a very obvious choice, since the market building (macellum) is inbetween the most significant buildings in the centre of the Civil Town of Aquincum. Nevertheless the results of those excavations, which took place here, were not comprehensively published, only preliminary reports or short artickles appeared. The need for a synthesis for this site urged the authors to re-evaluate publications, to search for manuscripts from bequests, to revise the available old excavation documentations, and to investigate the finds.

Chapter 2,3 and 4 of this book relates those more than 130 years of archaeological researches in the Aquincum Civil Town's macellum.

The row of the so-called 'old' excavations starts with the systematic excavations led by Bálint Kuzsinszky between 1882 and 1884 .
This $19^{\text {th }}$ century excavation can be considered quite modern, and he gave great, detailed descriptions of architectural phenomenons. His interest for architecture appears also in the presentation of the finds, as besides of a bronze statue's foot fragment, he mainly discusses lead- and carved architectural stone objects. He uncovered only the latest building phases of the macellum, but he connected many analogies to all the features he found. His publication is a complete report of the four year excavation carried out in the area of macellum.

The next research, executed in 1929 by Lajos Nagy identified the building as a macellum, and mentioned a pottery workshop and a bronze smithing workshop here, dating to the turn of the $2^{\text {nd }}-3^{\text {rd }}$ century to $240 \mathrm{AD}$. The confirmation of these workshops in lack of the original documentation is impossible, and the report unfortunately doesn't gives us enough information.

Melinda Kaba's 1960-1962 excavation took place as part of a large-scale conservation programme. It's main objective was to specify the building phases of the area and to define the correlation of the macellum with the other important, known buildings of the city.

The research in 1965 done by archaeologist Klára Póczy and architect Gyula Hajnóczy finally brought a breaktrough, as it clarified some incorrect ideas, and assessed that the macellum was still in use at the turn of the $3^{\text {rd }}-4^{\text {th }}$ century AD. They have found a kiln or oven, but it's relation to the hypothetical pottery workshop could not be confirmed.

The revise of the results of these four excavations was published in an MA thesis and two more articles written by Orsolya Láng in 2001, in 2003 and in 2007. This research, based on the prevoius documentation, has identified the building phases of the macellum in Aquincum Civil Town, and toroughly examined the tipology of macella. Epigraphical research also took place, which intended to identify the possible builder of the macellum.

Technically the research leans on three major sources: on the previous publications, on the original excavation documentations and on the find material. All these sources had their specific problematics. The main stumbling block for the authors was, that the previous four researches were not comprehensively published, only one out of four. Besides this, there were many missunderstandings and incorrect out- 
growths in the literature, so the publications as main source in this case must have been treated with care and with healthy critical sense.

The other possible source, the original documentation of the excavations was also problematic, because it was available only in two cases out of four. In addition these excavations were all carried out with different excavation methods using quite different standards.

Regarding the archaeological material difficulties occured as well. Finds were merely available for three cases out of four excavations, but the majority of the finds was accessible only for the last two campaigns. In addition those more than 50 years, which has passed since the last excavation, some objects were presumably mingled with other excavations' material, so the finds of this site were hard to identify.

The strenght of this work lies also in the fact, that the authors were needed to create their own methodology, or better said to find and apply those methods which allows in these restricted conditions to gather as much information, as possible, and to build up the coherent, well structured story of the macellum.

Despite the difficulties, researchers tried to find solutions to all the problems. Regarding the previous publications, the authors have checked the validity of all the published information, and we can have the detailed confutation or the contrary, the confirmation of every statement.

The second main source, the original excavation documentation was only available for the excavations in 1960-1962 and for the research from 1965 in the Archaeological Archives of the Budapest History Museum. The documentations were revised, and the data was postrecorded with modern, objective methods of archaeological documentation. In the matter of stratigraphy the Harris matrix was used, old photographs and plans were attached and re-evaluated, the different building phases got presented in consecutive ground-plans, and new conclusions were drawn.

The authors wanted in this book to present the available finds to make the analysis complete. The second part of the book focuses therefore on the possible pottery workshop (Chapter 5) and on the presentation of the finds (Chapter 6,7). At the end of the book a detailed catalogue of the objects can be found. The term 'finds' in this book mainly covers the wide range of ceramic objects, but glass, metal, worked bone objects, and different arhitectural elements (7.3), as well as the archaeozoological material (7.4).

Chapter 5 traces the exhaustive problematics of the possible pottery workshop, first attested by L. Nagy in 1929. In his publication he states the presence of a cellar and a well, and a series of vessels which he grouped by form. Unfortunately he did not describe in details the findings, no ground-plan survived, and the series of similar vessels can not be identified anymore in the inventory of the museum.

The idea of a pottery workshop appeared again in the course of the 1965 year's excavation, when a kiln/owen was unearthed. K. Póczy however considered that the ceramic finds attests rather a shop or a pottery storeroom, where the products of multiple workshops were sold, than a workshop in the middle of the city. The authors had shown, that by it's position in the stratigraphy, the owen is more suitable for baking, and it cannot belong to the presumed pottery workshop. The largest group of similar pottery can also be dated earlier, aproximately to the first half of the $2^{\text {nd }}$ century AD.

The possibility of a nearby pottery workshop after all can not be excluded, because finds (deformed pottery fragments, lamp mould, kiln furniture fragment) were discovered, which could indicate pottery production, but despite of pro arguments, the workshop could not be identified, further excavations would be needed.
As mentioned above, the work with the find material was problematic. Mainly the material of the last two excavations was used for this work (1960-62; 1965). It was clear from the beginning of the research, that objects found from the 1929 excavation are just a small part of the originally excavated material, and that the finds from 1960/62 and 1965 were hand-picked. Even so a conservative valuation and statistic was still possible. The publication finally deals with 717 vessels.

The methodology of pottery processing is noteworthy (Chapter 6). As in the Roman Period the pottery workshops of Aquincum used the same local Oligocene Kiscell type of clay for pottery production, and presumably similar techniques, the end products became very likely to each other. Therefore, the authors used a macroscopical analysis, which descends to particulars. By the macroscopical characteristics of the material 31 fabric groups were separated. The differences in colour, the categories of roughness, the grain size and frequency and the porosity of the material was defined for every object.

It was confirmed during the study of the ceramic material, that not only the form, but also different technological characteristics are indicating the different periods of production. Therefore the vessels were first sorted by material, slip, firing technique, than the occurence of forms was investigated within these groups.

Chapter 7 relates the wide variety of pottery finds, starting with the finds of the 1929 excavation. As expected, the material of the latest two excavations was way much varied. The following categories of pots are presented: thin walled/colour coated/coarse beakers, the handle fragment of an early Roman glazed patera, Terra Sigillata vessels and their imitations, imitations of Pompeian red wares, stamped PGW-s (Pannonische Glanztonware), Amphorae, vessels with namestamps, glazed and slipped pottery, the so called „Kiscell red wares”, which covers the very good quality redish pottery produced by the military pottery workshop of the Aquincum cannabae, flagons and jugs, the two and three handled vessels, coarse wares, and cooking wares. The publication shows an interest for the different types of bowls, which are quite varied: flanged bowls (Kragenschüssel), bowls with small flange (Leichtenschale, Ringschüssel, „Pátka” bowls), barbotine decorated bowls, S-profiled bowls, flattened hemispherical bowls, hemispherical bowls with beaded rims, and tripod vessels. The mortaria, caccabi, lids, strainers, turribula and storing vessels are presented in details as well. The categories of lamps and lamp-moulds, terracotta objects, ,lighthouse" pots and tiles are described and discussed here too. At the end of chapter 7 the glass, metal, bone and worked stone objects are also listed.

The authors intention is clear: they wanted to present the finds as detailed as possible, the complete collection in it's complexity, meanwhile the short history of vessel-forms is there as well, just like the dating and possible production place so we can track the route of almost every single potsherd from the pottery workshop to the Aquincum macellum.

The examination of the archaeozoological material has shown, that from the area of the macellum mainly the meaty parts of cattle, swine and large ungulates were discovered. These attes butchery activity the meaty parts were separated from those, which possibly were used for other purposes.

The last descriptive chapter of the book (Chapter 8) were separated summarizes the results of the old and new reserches. The emphasis falls on the coherent chronological reconstruction of the building phases of the area, completed with comprehendible ground-plan of every phase and with the evidence provided by the ceramic material. 
According to Chapter 8 the earliest signs of human activity are marked by some pits and a fossa, probably part of the southern part of a vilage-like settlement at the end of the 1 st - first decades of the $2^{\text {nd }}$ century AD.

Two wall fragments and filling layers belong to the second phase, possibly dating to the first half of the $2^{\text {nd }}$ century AD.

The remains of the third phase are marked with three walls, which could have been in use sometime during the middle of the $2^{\text {nd }}$ century AD.

Phase 4 is the period, when the presumed pottery workshop could have operated. The ground-plan of the same building is showing on the map. This has had most probably a commercial function. Seems, that it had been destroyed by fire around the middle of the $3^{\text {rd }}$ century AD

The building of the macellum was only erected in the $5^{\text {th }}$ phase at the middle of the $3^{\text {rd }}$ century, which corresponds to the last economical and architectural boom of the Civil Town. The remains of the previous phase were leveled, and a large building was constructed with a paved inner courtyard and with a tholos in its middle. Two rows of shops were opening to the neighbouring road and to the inner courtyard. Along the Western facade presumably a parking place for carriages was built. According to the archaeozoological investigations, meat-processing was one of the most plausible activities at this site in the second part of the $3^{\text {rd }}$ century AD.

In the last roman period (phase 6), between the end of the $3^{\text {rd }}$ beginning of the $4^{\text {th }}$ century $\mathrm{AD}$, the building of the macellum was possibly already in ruins. We have evidence only for the usage of the Western road and of a smaller building on the Western side of the investigated area.
The last phase (nr. 7) dates to the modern times, since refilled archaeological trenches and second world war bomb craters were documented.

The elegant aspect of the book is in concordance with it's content and as the work is written in english, the latest results of this research are accesible for foreign researchers as well. We have now a very accurate, traceable, well structured presentation of the history of the area and of the market-building, hence the macellum of the Aquincum Civil Town became a great source of analogy for the macella type of constructions in the province and in the whole Roman Empire.

From the point of view of pottery-researchers, one of the greatest merits of the book is, that it has proposed methodology for pottery processing which is actually based on the fastest and in the same time the most accurate macroscopical analysis man can make with a large quantity of ceramic material. Functional, useful illustration was added to it, just like recent literature. These features and the fact, that it represents such a large variety of finds, presented in such a detailed way, makes this book an excelent handbook for provincial pottery research.

One of the major objectives of this work was, to re-think and to sum up the accumulated information of the previous excavations. This effort draws the attention to a serious challenge in the archaeological research, namely to the urging need to reevaluate old excavations and their material. The authors of this book therefore are presenting us an elaborated methodology in this topic.

Ágota Ferencz-Mátéfi agotaferenczmatefi@gmail.com

\section{Editiones externae}

Nives Doneus (Hrsg.): Das kaiserzeitliche Gräberfeld von Halbturn, Burgenland. Mit Beiträgen von M. Berner, F. Daim, N. Doneus, E. Draganits, Ch. Ertel, H. Herold, G. K. Kunst, A. Preh, A. Rohatsch, H. Taeuber, U. Thanheiser, K. Vondrovec, H. Winter und S. Wiesinger Monographien des RGZM, Band 122, 1-4 Bände. Mainz 2014.

Die vorliegenden, das römerzeitliche Gräberfeld von Halbturn I ausführlich behandelnden vier Bände umfassen die multidisziplinären Endresultate der langjährigen Tätigkeit auf dem Fundplatz. Es ist eine methodologische Fortsetzung des im Jahre 2004 veröffentlichten Vorberichtes in der Reihe MFMA Bd. 10.

Die Gemeinde Halbturn liegt im nördlichen Burgenland (Bz. Neusiedl am See/A) in direkter Nachbarschaft der österreichisch-ungarischen Staatsgrenze. Geographisch schließt sie an die kleine ungarische Tiefebene an. Das Gebiet mit Siedlungs- und Grabfunden im Umland von Carnuntum wurde in der Römerzeit administrativ in Pannonia Superior integriert.

Im Vorwort (Teil 1, XIII) skizziert F. Daim die Rahmen des Halbturn-Projektes, an dem mehrere Disziplinen (Archäobotanik, Archäozoologie, Archäometrie, Geologie, Luftbildarchäologie und geophysikalische Prospektion) beteiligt waren. Die so erworbenen Erfahrungen bieten eine gute Ausgangsbasis für die zukünftigen Forschungsprojekte.

Das Rückgrat der Bearbeitung bildet das archäologische Material vom Gräberfeld Halbturn I, das im Jahre 2006 von Nives Doneus als Dissertation an der Universität Wien eingereiht wurde (Teil 1, S. 1-230)
Eine römerzeitliche Siedlungstätigkeit auf dem Gebiet der Gemeinde ist seit Langem belegt. Das sog. Gräberfeld II wurde im Jahre 1961 entdeckt und ist ohne Informationen über die gesamte Belegungsdauer in die erste Hälfte des 3. Jahrhunderts datiert. Mehr Bedeutung ist dem von 1988 bis 2002 systematisch und sogar vollständig freigelegten sog. Gräberfeld I aus mehreren Aspekten zugewiesen.

Die Verfasserin betont den ländlichen Charakter des Siedlungsgebietes, nämlich gehörte das Gräberfeld I zu einer Anlage von villa rustica, die sich in bestimmtem Sinne - wie „Grablage, -konstruktion, -ausstattung“ - von einem städtischen Milieu unterscheidet.

Im lang benutzten Gräberfeld- und Siedlungsareal könnten die Relikte der römerzeitlichen Flurparzellierung archäologisch nachgewiesen werden

Die Belegungsdauer des Grabareals reicht vom 2. bis ins 5. Jahrhundert, die eine gute Grundlage für die Überprüfung des Wechsels im Bestattungsritus und im Grabbrauchtum im Laufe der Jahrhunderte sowie für die Analyse der Frage nach dem demographischen Wandel, weiterhin nach der Zusammensetzung der Bewohnerschaft der nahe liegenden villa rustica bietet.

Die überraschend große Anzahl der Säuglings- und Kinderbestattungen ermöglichte eine detaillierte Behandlung der Frage nach dem sozialen Status der Kinder in der Gemeinschaft der Villenwirtschaft.

In der Einleitung (Teil 1, S. 1-2) schildert N. Doneus die Zielsetzung der Arbeit. Vor allem wurden die komplexe Veröffentlichung und die typochronologische Bewertung der Befunde und der Funde des Grabareals bestrebt. Daneben hat sie ein „Gräberfeldmuster“ durch die Analyse ,zur Organisation und der Variabilität der Bestattungen“ der einzelnen Perioden und durch die Vergleichsanalyse der 
verschiedenen Zeitphasen auszuarbeiten erzielt. Die Verfasserin betont die Bedeutung der anthropologischen Untersuchungen, die zu den Geschlechts- und Altersbestimmungen, den pathologischen Veränderungen und dem Gesundheitszustand der Gemeinde viele relevante Informationen beigeben konnten.

Im folgenden Kapitel umfasst die Verfasserin die Kenntnisse über den Fundort und die Fundgeschichte (Teil 1, S. 3-8).

Es wurden bereits während der Grabungskampagnen non-invasive Forschungsmethoden eingesetzt und wurden mit geomagnetischer Prospektion die Dimensionen des Gräberfeldes und der Siedlung untersucht und festgestellt. Es stellte sich heraus, dass das Gräberfeld und die Siedlung sich ans römerzeitliche Flurparzellensystem anpassen, dessen Spuren im Gelände gut nachzufolgen sind. Methodologisch ist hervorzuheben, dass die einfachen Grabgruben der Brand- und Körpergräber mit Magnetik nicht zu erkennen waren.

In Hinsicht der Landschaftsarchäologie wird es zur Kenntnis genommen, dass das Gräberfeld und die ca. 180 m östlich liegende Siedlung den geomorphologischen Bedingungen und den Bodeneigenschaften nach mit anderen siedlungsgeographischen Gegebenheiten zu bezeichnen sind. Im nächsten Abschnitt (Teil 1, S. 9-64) sind die Befunde einzeln betrachtet. Auf der gesamten Ausgrabungsfläche des Gräberfeldes von über $7000 \mathrm{~km}^{2}$ kamen zahlreiche Objekte zutage (Flurgräben, Grabgärtchen, Gräber, Gruben, Pfostengruben), wovon die größte Gruppe die Bestattungen ausmachen (ca. 160 Brandbestattungen und ca. 150 Körperbestattungen).

Von besonderer Bedeutung ist das mit geradlinigen Flurgräben markierte Gitternetz (Teil 1, S. 11) als Relikt der römerzeitlichen Parzellensystems. Die rechteckigen Parzellen sind ihrer Größe nach nicht einheitlich und der Meinung der Verfasserin nach passen sie nicht an das römische Maßsystem an. Bei der Beurteilung der Frage wurde unter den römischen Längenmaßen mit actus (von 35,52 m) gerechnet. Im äußersten Bereich des Gräberfeldes konnte eine Grundeinheit von 25 m ermittelt werden, für mehrere Parzellen gilt es aber auch nicht.

Diese Befundsituation ist einzigartig. Es wäre eine eingehendere Analyse des angewendeten (örtlichen) Systems bei der Aufteilung der Ackerparzellen aus der Sicht der römischen Agrargeschichte in der Zukunft sehr begrüßenswert. Aus den schriftlichen Quellen geht hervor, dass die Flurparzellen auf weitere rechteckige Stücke aufgeteilt wurden, sogar könnte eine Flur auf unterschiedliche Parzellengrößen mit den Längs- oder Querachsen aufgeteilt werden.

Das römische Gräberfeld hat die rechteckige, von den Gräben umschlossene Fläche von ca. $30 \times 145$ m völlig ausgenutzt. An der westlichen Seite hat es sogar die Parzellengrenze (Objekt 3) überschritten, die nach dem Errichten des Gräberfeldes wechselnd zugeschüttet und erneuert wurde, aber bis zur letzten Phase des Bestattungsareals als Orientierungslinie fungierte.

Die nächst behandelte Befundgruppe sind die ,,persönlichen Bestattungsräume“, d. h. die mit den „Gräbchen“ begrenzten Grabgärtchen, die von 1 bis 39 nummeriert sind (Teil 1, S. 14-45). Die Verfasserin hat von diesen Grabgärtchen - mit Ausnahme der ersten drei - aufgrund der Größe, der Form und der gleichen Lage vier künstliche Gruppen abgesondert (Grabgärtchengruppe 1-4) (Teil 1, S. 18). Die letzte inhomogene Gruppe wurde allein nach der Fundlage (westlich des Flurgrabens 3) gestaltet.

Im Folgenden sind die bestimmten Grabgärtchen ausführlich als Fundsituation beschrieben (Form, die Anzahl und die Lage der Brandund Körperbestattungen, die Orientierungsfragen usw.).

Bei einem bedeutenden Teil der Grabgärtchen ist nachweislich mit einem zentralen Brandgrab zu rechnen, dem weitere Brand- und Körperbestattungen angefügt wurden.
Unter den Befunden befindet sich die Analyse der Brandgräber mit geschätzter Anzahl (ca. 160) (Teil 1, S. 47-52). Es ist anzunehmen, dass die Brandgräber gerade wegen der Unsicherheitsfaktoren als „Objekt“ benannt und nummeriert wurden und allein die Körperbestattungen als Grab gekennzeichnet sind. Zu den Objekten als Brandgrab steht eine Konkordanzliste im Teil 3 (XI-XV).

Eine größere Konzentration der Brandbestattungen konnte im südlichen und nordwestlichen Bereich des Gräberfeldes beobachtet werden, im Norden beidseits des Flurgrabens (Objekt 3). Die Flurgräben blieben von den Brandgräbern unberührt (eine Ausnahme ist Brandgrab 1 im Flurgraben 3). Überschneidungen kommen innerhalb der Gruppe nicht vor. Den Mangel an Brandgräbern in einigen Gärtchen interpretiert die Autorin mit der Bodenerosion.

Im Fall von Materialvermischungen geht N. Doneus von einem ebenerdig angelegten gemeinsamen Verbrennungsplatz (oder Verbrennungsplätzen) aus. Bustumbestattungen sind nicht belegt. Bei den Brandbestattungen kommen die einfache Gruben, die Gruben mit Ziegelsetzung, die Urnen, die Stein- und die Holzkisten ebenfalls vor.

Die Körperbestattungen mit einer Anzahl um 150 sind unregelmäßig verteilt. Anhand der Gräberfeldkarte ist aber eine Konzentration westlich des Flurgrabens 3 zu beobachten (Teil 1, S. 53-60). Die Orientierungen und die Grabkonstruktionen (einfache Gruben, partielle Steinsetzung, Spolien, Sarkophag, mit Ziegelsetzung und Ziegelplatten, Holzsärge) sind vielfältig. Mehr als ein Viertel ist nach den Flurgräben ausgerichtet (NNW-SSO). Aus chronologischen Gründen ist der Erscheinung, dass mehr als ein Viertel der Körperbestattungen in die Flurgräben und Gräbchen hingelegt ist, besondere Bedeutung zuzuschreiben. Letztendlich sind die sonstigen Befunde (Gruben und Pfostenlöcher) erläutert.

Das folgende Kapitel dient zur Behandlung der Funde nach den Warengattungen. Aus der Sicht der Chronologie müssen die TS-Funde extra erwähnt werden. Das einzige südgallische Produkt stammt aus dem Grubenhaus (Objekt 250) in sekundärer Lage. Daneben sind weitere Produkte aus Mittelgallien, Rheinzabern, Westerndorf und Pfaffenhofen vorhanden. Von den 55 Stücken von mittelgallischer Provenienz kamen 9 Stücke als Beigaben der Brandgräber vor. Von den 113 Rheinzaberner Stücken (die Gesamtzahlen können anhand der Tabelle zusammengerechnet werden; Teil 1, S. 228-230) wurden 42 als Beigaben der Brandgräber bekannt. Von 16 Westerndorfer Produkten wurden 5 ins Grab gelegt, während unter den 23 Pfaffenhofener Gefäßen kamen 13 als Beigaben vor. Die Verteilung der TS-Bruchstücke auf der Gesamtfläche wird nicht ausführlicher interpretiert. Allein bei den Westerndorfer und Pfaffenhofener Waren wurde auf die gleiche räumliche Verteilung hingewiesen. Anhand der Verteilungskarten sind manche Verschiebungen zwischen den Produktstätten nachvollziehbar.

Die chronologischen Rahmen des Auftretens der spätrömischen glasierten Ware sind öfters diskutiert, eine mögliche Datierung vom Ende des 3. Jahrhunderts wird aber immer mehr angenommen. Glasierte Ware als Bestandteil der spätrömischen Körpergräber ist eine allgemeine Erscheinung im pannonischen Raum. Was diese Warengruppe betrifft, modifiziert die Veröffentlichung von Halbturn unsere bisherigen Kenntnisse in manchen Bereichen. Glasierte Gefäße waren in den Brandgräbern bis jetzt nicht belegt, in Halbturn kamen aber sogar drei Reibschüsseln vor (Taf. 29.2, 236.6, 300.1). In den Körpergräbern von Pannonien ist das Vorkommen der glasierten Reibschüssel - entgegen den anderen funktionellen Formtypen - eine seltene Erscheinung. Eine der frühesten Vertreter der Reibschüsseln mit Glasur und roter Bemalung stammt aus einem Brandgrab, die andere aus einem Körpergrab (Grab 49) mit Constans I.-Münze aus dem Jahr $337 / 340$ wird terminus post quem datiert. 
In den darauffolgenden Kapiteln werden die chronologischen Fragen und die Bestattungssitten zusammengefasst.

Der Untersuchungszeitraum beginnt im 2. Jahrhundert und dauerte bis in das 5. Jahrhundert an. Die Gründung von villa rustica und die Parzellierung ist früher einzusetzen als die Errichtung des Gräberfeldes Im Gräberfeld ist eine kontinuierliche Belegung nachgewiesen, die in drei Hauptphasen (2. und 3. Jahrhundert, 4.-5. Jahrhundert) unterteilt wurde. Die Verfasserin versuchte die nach bestimmten Kriterien zusammengesetzten Grabgärtengruppen (Kapitel „Befunde“, Teil 1, S. 18) mit der Relativ- und Absolutchronologie des Gräberfeldes zu korrelieren.

In Klammern ist anzumerken, dass auf der Seite bei den „Ältesten Grabgärtchen“ ein kleiner materieller Fehler auftritt: Anstatt ,mittelgallische Terra Sigillata" sollte südgallische sowohl im Text als auch in der Fußnote stehen.

Relativchronologische Reihenfolge: Grabgärtchengruppe $1 \mathrm{mit}$ massivem Vorkommen von Lezoux-Produkten nach der Mitte des 2. Jahrhunderts, Grabgärtchengruppe 2 mit zentralen Brandbestattungen mit Rheinzaberner Dominanz. Für das 3. Jahrhundert wurde eine räumliche Ausbreitung in alle Richtungen registriert. Im Norden: Grabgärtchen 1-3, im Westen: Grabgärtchengruppe 4, im Süden: Grabgärtchengruppe 3 (erstes Drittel des 3. Jahrhunderts mit TS-Produkten von Rheinzaberner und Westerndorfer Provenienz), die - mit Ausnahme des Südareals, dessen Aufgeben die Autorin in das letzte Drittel des 3. Jahrhunderts setzt - bis zur Endphase der Brandrituale, d. h. bis zum frühen 4. Jahrhundert, genutzt wurde. Die jüngste Brandbestattung ist mit einer Zwiebelknopffibel Pröttel Typ 1 B (Objekt 362) - mit einer abgenutzten Münze aus dem Jahr 305 - datiert.

Im 4. Jahrhundert ist ein räumlicher Schwerpunkt westlich des Flurgrabens 3 zu registrieren. Um eine chronologische Entwicklung innerhalb der spätrömischen Phase mit Körperbestattungen zu rekonstruieren, versucht N. Doneus anhand der Keramikbeigaben und der räumlichen Lage der Bestattungen eine Gräbergruppe zu bilden. Die aufgebaute chronologische Reihe basiert hauptsächlich auf den Keramikbeigaben, deren Rolle in der Feinchronologie in manchen Punkten diskutiert werden könnte. Die eindeutige Zuweisung der Gräber mit Keramik von polierter bzw. eingeglätteter Oberfläche ins späte 4. bzw. 5. Jahrhundert muss anhand der sonstigen pannonischen Beispiele detaillierter betrachtet werden.

Anschließend diskutiert die Verfasserin die Bestattungssitten im Laufe der Jahrhunderte: zuerst die Befundsituation, dann die Frage nach der Grablage und nach dem Zeit- und Materialaufwand. Die Grabgärtchen als familiäre Bestattungsräume haben ihre Bedeutung nach einer Epoche verloren. Es wurden dann die jüngeren Brandbestattungen in die Umfassungsgräbchen hingelegt. Im 3. Jahrhundert hatte man immer mehr die freien Flächen außerhalb der Grabgärtchen benutzt. Das Phänomen, dass die Säuglinge in den Umfassungsgräbchen ohne Brandritus beerdigt wurden, bietet wichtige Informationen über den Totenkult der Römer.

Die mit Erdhügeln gekennzeichneten Grabgärtchen hält die Autorin für eine einzigartige Erscheinung in Halbturn. Eine vergleichende Untersuchung mit den sonstigen Teilen Pannoniens (so z. B. die Grabgärtchen und die Hügelgräber mit Umfriedung) ging leider über den in der Publikation erörterten Bereich hinaus.

Als ein örtliches Spezifikum ist die Verwendung der Gefäßböden als Lampe seit dem Ende des 3. Jahrhunderts sowohl in den Brand- als auch in den Körperbestattungen zu beobachten.

Im folgenden Teil ist eine Synthese (Chronologie und Brandsitten) erzielt. Es sind alle Gräber und sonstige Befunde ihrer räumlichen und chronologischen Lage nach in Bezug auf das Ganze des Gräberfeldes in sechs Gruppen unterteilt (I-VI) (Teil 1, S. 180-191). Die Gruppierung basiert auf den chronologischen Resultaten, die Nummerierung folgt aber nicht der chronologischen Reihenfolge.
Gräberfeldgruppe I (rechteckige Umfriedung im nördlichen Areal): erstes Drittel des 3. Jahrhunderts bis ins das 4. Jahrhundert

Gräberfeldgruppe II (Grabgärtchengruppe 1, Grabgärtchen 2 und 3): zweite Hälfte des 2. Jahrhunderts - Anfang des 3. Jahrhunderts

Gräberfeldgruppe III (Grabgärtchengruppe 2 mit Ausnahme des Grabgärtchens 9): letztes Drittel des 2. Jahrhunderts - Anfang des 3. Jahrhunderts

Gräberfeldgruppe IV (Grabgärtchengruppe 3): 3. Jahrhundert

Gräberfeldgruppe V (Grabgärtchengruppe 4): Ende des 2. Jahrhunderts - erstes Drittel des 4. Jahrhunderts

Gräberfeldgruppe VI (Körperbestattungen): 4.-5. Jahrhundert

Dieses System modelliert eine räumliche Verbreitung des Brandgräberfeldes vom Norden nach Süden. Das Gräberfeld mit Körperbestattungen des 4.-5. Jahrhunderts konzentriert sich hauptsächlich auf einem früher nicht benutzten, westlich des Flurgrabens 3 liegenden Areal. Dieses Bild modifiziert in vielen Punkten die im Vorbericht im Jahre 2004 von A. Stuppner veröffentliche chronologische und räumliche Situation (Halbturn I. Das kaiserzeitliche Gräberfeld und die Villa von Halbturn, Burgenland. Naturräumliche Voraussetzungen, Prospektion und Vorbericht. Hrsg. von F. Daim und N. Doneus. Monographien zur Frühgeschichte und Mittelalterarchäologie 10. Innsbruck 2004).

Das archäologische Kapitel ist einleuchtend aufgeteilt, aber eine derartige Gliederung führt zu häufigen Wiederholungen, während manche nötigen Informationen in mehreren Passagen, gelegentlich weit voneinander entfernt behandelt werden.

Im darauffolgenden Aufsatz des ersten Bandes wurde das aus dem Kindergrab 147 zum Vorschein gekommene jüdische Goldamulett, das griechisch beschriftet ist, von H. Taeuber ausführlich dargelegt (Teil 1, S. 231-235). Es ist ein wichtiges weiteres Zeugnis für die Diaspora-Juden im pannonischen Bereich aus der Epoche am Ende des 3. Jahrhunderts bis in das 4. Jahrhundert.

K. Vondrovec und H. Winter behandeln die Münzfunde (Teil 1, S. 237-254). Von den 45 Münzen könnten 23 Stücke den Körpergräbern zugewiesen werden. Am Ende des Kapitels steht eine Konkordanzliste, in der jene Münzen mit archäologischer Datierung der Befundsituation überprüft sind. Anhand des methodischen Ansatzes der Autoren, ,,aus der archäologischen Datierung Rückschlüsse auf ein Umlauf- bzw. Gebrauchsverhalten von Münzen zu ziehen, anstatt umgekehrt Befunde nach Prägezeiten von Münzen zu datieren“, sollte die Datierungszuverlässigkeit der sonstigen pannonischen Grabmünzen in einzelnen Etappen pro und contra argumentiert werden.

Ch. Ertel hat von den insgesamt 28 sekundär verwendeten Steinartefakten (Spoliengräber des 4.-5. Jahrhunderts) eine Grabädikula von der Mitte des 2. Jahrhunderts rekonstruiert, die ursprünglich auf dem Gräberfeldareal stand (Teil 1, S. 255-276).

Im Teil 2 haben sich A. Rohatsch und E. Draganits (Teil 2, S. 691-716) mit Herkunftsbestimmungen der Steinfragmente beschäftigt, wobei sie zum Ergebnis kamen, dass die Mehrheit der Steinmaterialien aus dem Umfeld von etwa $20 \mathrm{~km}$ stammt.

Im Abschlusskapitel von Teil 1 steht ein methodischer Versuch von E. Draganits und A. Preh (Teil 1, S. 277-298), wie die Volumina der Umfassungsgräbchen ausgerechnet werden könnte. Es führt zu Überlegungen über die Verwendungszwecke des Erdreichs aus den Gräben und zur Berechnung der Höhe und Breite von Grabhügeln. Diese Modellberechnungen stehen als wichtiges technisches Hilfsmittel für weitere ähnliche Analysen zur Verfügung.

Im Teil 2 sind die naturwissenschaftlichen Behandlungen veröffentlicht.

M. Berner hat die demographischen und paläopathologischen Charakteristika des Skelettmaterials des birituellen Gräberfeldes eingehend bearbeitet. Sie gibt einen guten Einblick in die ländliche Gemein- 
schaft (Geschlechtsverteilung, Sterbealter), in die demographischen Änderungen - die Zahl der Individuen aus dem 2.-3. Jahrhundert ist um ein Vierfaches höher als die Bestatteten aus dem 4. und 5. Jahrhundert - sowie in den Gesundheitsstatus und die Lebensgewohnheiten der Bevölkerung. In Halbturn sollte eine im provinzialrömischen Bereich einzigartige Erscheinung extra erwähnt werden: Von insgesamt 317 Gräbern könnten 121 den Säuglingen und Kleinkindern zugezählt werden ( 85 unter einem Jahr, wovon 75 weniger unter halbem Jahr). Am Ende der anthropologischen Bewertung steht getrennt ein Katalog der Brand- und der Körpergräber.

Darauffolgend analysieren G. K. Kunst die Tierreste (Teil 2, S. 485-640) und U. Thanheiser in Zusammenarbeit mit S. Wiesinger die Pflanzenreste (Teil 2, S. 641-664) aus dem Gräberfeldbereich.

Die petrographische Dünnschliffanalyse von $\mathrm{H}$. Herold an den 32 Keramikproben und 8 Tonproben aus der Umgebung haben zu folgenden Ergebnissen geführt (Teil 2, S. 665-690): Die grobkörnige graue Keramik wurde vom 2. bis ins 5. Jahrhundert kontinuierlich hergestellt; die Rohstoffe standen für alle erfassten Keramikgruppen lokal zur Verfügung; weiterhin stimmt das Halbturner Material mit manchen Keramikgruppen von Carnuntum überein.

I. G. FARKAS The Dislocation of the Roman Army in Raetia. BAR International Series 2723. Oxford 2015. ISBN 978-1-40731378-8 534 p. 80 fig.

The work of more than a half thousand pages offers much more than it is suggested by the title, as apart from historical questions of the Roman army of Raetia, it deals with the geographic and ethnic layout of the province before the Roman invasion in detail, as well as with the summer campaign of $15 \mathrm{BC}$, considered as the conquest of the province, and also with its organisation and administration. The work analyzes the antique sources on Raetia with philological accurateness, and discusses in depth the issue of Raetia-Vindelicia, present predominantly in the early sources.

One of the most important precedents of the work is the 8-page article of H.-J. Kellner on Exercitus Raeticus, published in the 1971 volume of Bayerische Vorgeschichtsblätter, but its methodology was also impacted by J.E.H. Spaul's book from 2000 on the history of the auxiliary units of the Roman army, by the publication of G. Alföldy on the auxiliary troops, and by the article of H. Wolf on the army of Raetia published in 2000. As one can observe, the topic already had a rich secondary literature by the time the author started his research relying on a novel methodological approach.

The work consists of four greater units. The first gives a clear and sufficient overview of the Raetian camps - this part could be used as a foundation to discuss in detail the question of dislocatio, and the author could locate here the introduction of troops recorded by their diploms, inscriptions and instrumenta findings as well. This part of the book contains four further chapters, presenting legionary fortress, auxiliary and smaller forts - the so-called numerus-sized military establishments. The latter term refers solely to the size of the troops, as actual numerus corps were never stationed in Raetia. The second greater part presents the history and camp sites of units stationed in the province. The third, summarizing part deals with the question of the occupation of Raetia, and with the military history of the province. This latter topic is divided into eight periods: the ages of the Augustan and Julio-Claudian dynasties, the decades of the Flavians and Nerva, the reign of Trajan, ages of Hadrian and Antonius Pius, the reign of Marcus Aurelius before the Marcomannic wars, the decades of the
Im Teil 3 (Objekt 1-367) und 4 (Objekt 368-504, Grab 1-182) wurde der Katalog der Befunde veröffentlicht: zuerst die Objekte (einschließlich der Brandgräber), dann die Gräber (Körpergräber) in nummerierter Reihenfolge mit den Tafeln des archäologischen Materials und den Zeichnungen der Fundobjekte.

Diese monumentalen Bände behandeln eine außerordentliche Fundsituation eines langlebigen und vollständig ausgegrabenen Gräberfeldes von einer Villenwirtschaft. Sie lassen nützliche Erkenntnisse über die Bestattungsriten der weniger bekannten Periode des 3. Jahrhunderts gewinnen. Dem Brandritus konnte bis ins 4. Jahrhundert nachgefolgt werden. Die ersten Körpergräber werden - abgesehen von den Kinderbestattungen - erst ins erste Drittel des 4. Jahrhunderts datiert. Die multidisziplinäre Bearbeitung des Fundplatzes steht als ein Musterbeispiel für weitere Untersuchungen zur Verfügung.

Friderika Horváth Archäologisches Institut der Ungarischen Akademie der Wissenschaften Úri utca 49, H-1014 Budapest, Ungarn Horvath.Friderika@btk.mta.hu

Marcomannic wars, and the age of the Severan dynasty until the fall of the Raetian limes in 254. Altogether, 120 charts and maps illustrate the discussions of the work. A smaller subchapter deals with the information available regarding the religion of the army, and with the soldiers of different ranks from the rank of legatus to private soldiers and even to veterans; the authors discusses the possibilities for mobility within the army, promotions and construction works of the troops. The fourth great part of the work consists of a register of selected inscriptions concerning legions and auxiliary troops, of data from auctors about Raetia, and of military diplomas. The work is completed by a rich bibliography.

With regard to the description of forts, as a result of new available methods the number of known military constructions significantly grew in the recent decades. Before that, particular limes establishments could only be known by excavations and field surveys, and also with the help of surveys and documentational activities started in the 1890s. In the latter process, groundbreaking merits to be attributed to the Reichs-Limeskommission, founded by Th. Mommsen in 1892, an organisation that fulfilled its job in a framework provided by a research institution. New methods allowing for devastation-free research considerably increased the number of known military constructions: the aereal photographs of $\mathrm{O}$. Braasch, geophysical and magnetometer researches of E. Faßbinder, dendrochronological measurements of W Czysz and others resulted not only in the recognition of more than thirty new limes establishments, but also contributed to the correct dating of the objects. Opportunities offered by new methodologies were extensively used by the author, he refers to them at the discussions of particular camps - still, the work lacks a summary, a jointed list of castella and numerus-scale camps that could be observed with the help of aereal photographs or other interdisciplinary methods.

The first problematic point of the work is the evaluation of the archaeological site of Augsburg-Oberhausen, which is enlisted among legionary fortresses, however, in the short description the author avoids to entitle the site as legionary fortress. The only known excavation on the site since 1911 was conducted by P. Reinecke in 1913. In the past hundred years, generations of archaeologists made an attempt to the interpretation of the original function of the site. While Drexler regarded it a camp, the excavator P. Reinecke was careful enough to 
use the term "Waffenplatz". On the basis of findings, that consists of coins, pottery and metal (brooches and arms) items, there is no doubt that the Augsburg-Oberhausen site can be dated to the Augustan period. According to the analyses of S. von Schnurbein, the group of artifacts can be dated to between 8/5 BC and $\mathrm{AD} 9$. Based on the Weisenau-type helmet and typical armament, such as the pilum or stylae and daggers, it is also obvious that legionary soldiers were stationed at this base, along with auxiliary units. Even if we disregard such seldomly accepted ideas as the concept of W. Hübener, that suggests that the site might have had a sacrificial function and belonged to Celtic tribes, based on the evidence referred above, it is obvious that interpreting Augsburg-Oberhausen as a legionary fortress overly simplifies this very complex problem.

The second legionary fortress is Eining-Unterfeld, there are no controversies about this in scholarship. Legio III Italica might had been stationed here between 165/66 and 179, yet before they were getting transferred to the permanent fortress in Regensburg. The case of legio II Italica, a unit that was created also in the age of Marcus Aurelius was somewhat similar, as in the beginning they were stationed in Albing before they were transferred to their fortress in Lauriacum. Based on epigraphic evidence, T. Fischer dated the erection of the legionary fortress in Eining to 172. This is opposed by the author with the help of coins and terra sigillatae, and the date 165/66 is suggested instead. However, samian cannot be dated with such accurateness, therefore I would suggest the question of Feindatierung to be left open. Thus, after the age of Marcus Aurelius, Raetia was not a provincia iners any more; from 179 on, the legion was stationed in the fortress at Regensburg, and in this manner, they could participate in the construction of auxiliary forts, such as of Böhming or Ellingen. In point $B$ of this part, the author presents 45 auxiliary forts. In 9 of these - ala, in 23 - cohors, and in 10 - cohors equitata were stationed.

From the Augustan age, we have knowledge of one single auxiliary fort only, the camp in Friedberg-Rederzhausen, discovered by O. Braasch, excavated and published by S.von Schnurbein. Regarding its size, the military establishment corresponds to later cohors forts, however, its garrison is unknown. The aereal photographs show two castella, which presses me to raise the question - could one of these serve as a training camp?

The volume presents auxuliary forts in a chronological order; first, it discusses briefly the establishments of the Tiberian-Claudian age. These are investigated with the help of unified aspects: first, their layout is recorded, then they are compared to surrounding fortresses, which is followed by the determination of their size (and of the types of troops they could house), their surface, their time of construction and later transformations. Whenever it is possible, the author provides a plan based on available publications, and a list of literature on the camp along with a register entitled "further reading", enlisting publications that deal with the given fortress only partially (e.g. as a parallel).

As it is known since the work of G. Ulbert, Aislingen is regarded as the earliest of auxiliary forts, having been built under the reign of Tiberius. In my view, Aislingen, and probably Oberstimm as well, was part of a wider strategical concept involving the entire ripa Danuvii. The inscription at the gorge of the Iron Gates, recording the road construction activities of the Moeasian legions, is from 33/34 AD - the road provided transport between the lower and the upper sections of the river Danube. A road of timber structure, carved into the rocks, implies the existence of a permanent garrison, especially if we consider the neighboring Dacians. In forts along the lower Danube around the Iron Gates (Djerdap), we may find the same L. Gellius pottery (Transdierna, Boljetin, Ratiaria, Montana, Oescus) as in Aisling, or in Lentia or Carnuntum in the Tiberian age. Thus, these sites located at the end points of radially running roads are indicators of the first Danubian forts. In this manner, Aislingen may be regarded as part of a permanent Danubian fort system, the construction of which might have taken place in the same period when all the above mentioned forts were erected as part of the same conceptual framework. The book introduces 10 auxiliary forts from the Julian-Claudian age altogether in detail: among them, the castella of Burghöfe and Oberstimm are the most well-exploreded ones, their erection can be dated to 43/45. Even though the camps from the Claudian age were abandoned - similarly to Ladenburg - the majority of the vici were inhabited in later times, such as in the cases of Burgheim or Risstissen, while Burghöfe even became part of the $4^{\text {th }}$-century defence system. In the case of Oberstimm, already $\mathrm{H}$. Schönberger called attention to the lack of military establishments from the Claudian-Flavian age in the Eastern direction between Oberstimm and Linz. One of the reasons behind this might obviously be that in this area the other side of the Danube was inhabited by the allied Hermunduri, who could visit the colonia splendidissima independently (this site was probably Cambodunum).

The number of forts in this section, built under the Flavians and Nerva, counts 9. Several of these were built north from the Danube already (e.g. Gomadingen, Kösching), or in a manner that allowed the fortress to serve as a crossing point on the river (e.g. Günzburg). Along the eastern section, the constructions provided substitution for the lacking links in the defence line (Künzing, Straubing, Passau I). Among these forts, the most prominent is the one in Eining, where geophysical surveys were also implemented in the recent years. In the south-eastern corner of this camp, a new, small fortification was built in the $4^{\text {th }}$ century. This type of construction work is also well known from Pannonia - let us think about the small forts in Carnuntum or Azaum.

The identification of garrisons in particular forts is not an easy task in many cases, and on the basis of a single stamp on tile, the identification is severely dubious. Although the late Roman Period is not investigated by the author, based on the above mentioned layout features and the continuous presence of garrisons in the same place in certain cases, the connection of earlier and later periods is apparent. In the case of Passau I for instance, the data of Notitia Dignitatum about the cohors nova Batavorum might be pertained to the coh. IX Batavorum that was stationed there earlier. One of the most well-researched, and within the framework of a monograph, most elaborately analyzed forts is the one in Regensburg-Kumpfmühl, expanded under Hadrian. Is it possible that this extension is related to the transfer of an other troop to the camp? Based on epigraphic data, between the decades $80 \mathrm{AD}$ (earlier, A. Faber dated the construction of the camp to the year of the four emperors) and the Marcomannic wars, at least 3 units were stationed in this camp. Its destruction is dated to 170 . This was the same year when Pannonia suffered the destruction of most of its forts. The same fate awaited the castellum of Straubing III. According to T. Fischer, a late Roman fortress was built also in Straubing in the $3^{\text {rd }}$ century.

The number of forts from the age of Trajan is 19; with a few exceptions, all of them were built north from the Danube. Burladingen was built on the western section of the Alblimes - this camp was dated to the 80 s by Heiligmann, but as it turned out from the dendrochronologic analyses carried out by S. Sommer, it could not have been built before Trajan.

Among the Hadrianic-Antonine forts, it is the one in Pfünz where considerable research had been carried out already in the years 1884-1890. Earlier, this castellum was regarded to originate from the Flavian age, but according to its inscription referring to the constructions, it can be dated to the reign of Hadrian. 
What concern the forts from the age of Marcus, the one from Aalen can be dated to the 160 s, on the basis of dendrochronologic analyses and its building inscription as well. It is probable that the fort in Aalen was also responsible for the defence of the limes section between Schirenhof and Kelheim. However, the origin of its modern name from the word ala is uncertain.

The primary assignment of numerus-sized camps might have been the control of roads. In the case of Burlafingen, the Hagenautype helmet permits the author to raise the possibility that one of the corps of legio XVI Gallica remained in the province yet after the transfer of the main forces. M. Mackensen published a small monograph on the recently discovered camp in Nersingen. A monograph was published by W. Zanier on the fort of Ellingen, built in the age of Hadrian. However, it is not known whether this small fortification was destructed in the Marcomannic wars or in another period; according to an inscription from the year 182, renovations or fortifying works were carried out here. As it had been proposed by H.-J. Kellner, it is without doubt that the Marcomannic wars destructed Böhming; a burnt layer and an inscription from 181 recording the restoration provides evidence concerning previous destruction.

This chapter also contains the description of 36 smaller fortifications that previously were regarded burgi, and the chapter is also extended with the research history and summary of construction periods of the Limestor in Dalkingen. The small fort in RegensburgGroßprüfeningen was known by aereal exploration conducted in the 1990s. As numismatic finds give evidence, the small forts, built after 179 , was devastated after $242 / 43$. The research of sigillata, however, refused to accept this dating, although its further usage, propagated also by the author, cannot be excluded. It remains an unsolved question, however, if the fort was rebuilt after the German invasion in 254.

The volume offers a notable solution to the chronological issue of the small fort of Weltenburg-Cadget. While T. Fischer assumed a Claudian construction, the author suggests the end of the 1st century as the date of its creation.

With the help of data from the literature, epigraphic evidence and instrumenta inscriptions, the second part summarizes knowledge concerning the history of particular troop units.

From among the legions, the author comments on the units stationed in Raetia after the summer campaign of the year $15 \mathrm{BC}$, participating in the campaign of Drusus. Early legio-vexillations could remain in the province perhaps until AD 41 or 68, probably exactly in Augsburg-Oberhausen or Burlafingen. The province was a provincia iners until 165/66. Legions were stationed here only after the establishment of legio Italica II and Italica III. After P. Kovács, the author encounters the arguments and counter-arguments (e.g. the plans of creating the provinces of Marcomannia és Sarmatia) concerning causes of the establishment of the two legions. The second legion, the soldiers of Augusta III were fighting in Raetia already during the Marcomannic wars. As the legion supported Maximinus Thrax during his attacks in Aquileia, this corps was punished by Gordianus III. The location of their fortress might have been Burgsalach, or according to S. Sommer, Faimingen II.

Many of the auxiliary troops were later stationed in Pannonia, such as the ala I Hispanorum Auriana (after 69, in the camp of Aquincum-Víziváros), the ala I Thracum veterana after 106, the coh. III Batavorum after 118/119, and the coh. III Thracum veterana between 84 and 106/119. Contrarily, the coh. I Breucorum was created after the repression of the Pannonian-Dalmatian revolt, and it was stationed in Germania at first, before it was moved to Raetia. In the descriptions of the history of particular troops, the author gives a detailed analysis of the circumstances of the formation of units, and he also describes their station sites. He provides a table of considerable sources and the location of inscriptions in the case of each troop, that offers a great support in the identification of the basis of the corps.

The tile stamps of coh. I Flavia Canathenorum are present equally in Eining, Regensburg-Kumpfmühl, Kösching and Straubing. Is it possible that this cohors was a technical unit (as the coh. VII Breucorum in Pannonia), the stationary base of which is unidentifiable on the basis of tile stamps? In the case of coh.VI Lusitanorum, the author supposes that either the person who issued one of the diploms, or the engraver made a mistake while noting the number, and in fact, the dismiss document was given to a soldier of VII Lusitanorum. This method had been frequently applied, for instance, in the mile data of itineraria, if the distance did not correspond to the source data. Still, I have a doubt that during the argumentation of the ordinal number of troops, this method may lead to a deliberate exclusion or inclusion of troops into the army of the province, solely because the scribe made a mistake noting the ordinal number.

The differentiation of troops with similar names is indeed brilliant - e.g. in the case of coh. III Thracum c.R., as inscriptions not always provide the correct names of units. Certain troops can be included in the exercitus Raeticus only on a logical basis. For instance, an inscription mentions coh. Truplinorum, the only noted thing about which is that the Trumplini are enlisted on Tropheum Alpium among the defeated Raetian tribes. The tight interconnection of the history of particular units and the history of forts constructions is well illustrated by the transfer of coh. IV Tungrorum vexillatio to Mauretania Tingitana, that resulted in leaving behind the fort in Faimingen. There is no trace of further units in the epigraphic material, supposedly a part of them was resident in Raetia only at the time of the occupation, such as legio XIII Gemina XXI Rapax or legio XIX.

The summarizing part deals with the geography and the ethnical relations of the province before the conquest, based on data taken from Strabon, Plinius, Ptolemaios, Tacitus and Dio Cassius. Archaeological evidence is scarcely applicable in this issue. Antique authors had relatively small knowledge about this area, thus in many cases, the lack of information is supplemented with mythology. The author claims that besides its military importance, the conquest of Raetia was an economic interest, as the areas north of the Alps were reknown for their good quality soil. The author deals in detail with the campaign of year $15 \mathrm{BC}$ that resulted in the conquest of the province. This was preceded by the annexation of regnum Noricum.

Numerous fortress participating in the campaigns were identified already by fortress - e.g. Dangstettent (here a reference to Fingerlin, who carried out the excavations, would be more appropriate). As H.-J. Kellner argued, the organization of Raetia as a province was implemented half a century later, when the first governor of the province was appointed. In the past decades, the question of organizing the province was in the focus of scholarship. According to P. Kovács, the redactio in formam provinciae might had taken place in the age of Tiberius. In 2001, R. Rollinger and A. Schaub, independently from each other, claimed that the data of Velleius Paterculus - Raetiam autem et Vindelicos ac Noricos Pannoniamque et Scordiscos novo imperio nostro subiunxit provincias - should be taken as in the time of the publication of the work, 30 AD, Raetia and Pannonia were provinces in effect, perhaps already with functioning public administration.

The book discusses in detail with the question of the dichotomy of Raetia-Vindelicia, and makes an attempt to locate the two ethnic groups geographically. The term Vindelicia-Vindelici is present predominantly in early sources - relevant data is presented in charts as well. From the later sources, Vindelici is mentioned only by Claudianus, but it is likely that the data concerns Vandalicis, similarly to the case of Geti and Gothi. 
The chapter of the summary concerning the military history of Raetia is excellent. The chapter sums up available information regarding the movements and transfers of troops, the construction, renovation and abandonment of forts. Not much is known about units of the Augustan age, which can be a result of the feature of the army that its main task was to provide inner security instead of defence from an outer enemy. In this job, they could count on the inhabitants of civic settlements: residents of Kempten, Auerberg, Lorenzberg bei Epfach supported the Roman rule as well. Withdrawal from Augsburg-Oberhausen is dated to the age of Claudius, but it can be supposed that units stationed here were retreated already in $9 \mathrm{AD}$, after the defeat in the battle of Teutoburg.

In the age of the Julian-Claudian dynasty, already 10 forts and 4-6 smaller strongpoints were built along the Danube. There is a fine, chronology-based chart presenting the station camps of particular groups and their transformations, the related sources and the chronological order of forts. A map from each period presents the fortresses of the limes, the territories controlled by the Romans, the new establishments and abandoned forts. Although more than a hundred years have gone since the publication of the ORL volumes, chronological questions remained open in many cases. Following the ideas of S. Sommer, the author supposes that Trajanic constructions were carried out not at the turn of $1^{\text {st }}-2^{\text {nd }}$ centuries, but presumably in the years after the Dacian wars. Numerous new, smaller strongpoints were built in the age of Marcus Aurelius, before the Marcomannic wars; on the basis of dendrochronological data, bridges were also built in this period. The construction of a bridge in Intercisa was carried out roughly in the same age. The most important step of the reorganizations after the wars was the construction of a permanent legionary fortress in Regensburg. In his period, half-circle shaped towers emerged as new elements of layout plans: similar ones in Pannonia can also be dated to the age of Commodus (Quadrata, Campona, Matrica). Between 238-253 AD, the legion Augusta III was also stationed in the province. The author presents informatively the gradual desertion of forts of the Raetian limes and their destruction in 254. Earlier, the abandonment of the northern part of the province was related with the invasion of the Alemanns in 233, and with the events of 241/42. Opposed to Dacia, Raetia was not evacuated according to a plan, but it was the destruction of 4 ala and $9 \mathrm{co}$ hors in the eastern campaign of Valerianus that was proven to be fatal for the province. In the last will of Augustus, in his admonitions to his successors, the violation of coercendi intra terminos imperii concerned exactly those provinces that were conquered by the successors east from the Rhine and north of the Danube. These had to be abandoned and evacuated by the third quarter of the $3^{\text {rd }}$ century.

The chart presenting military constitutions, camps and troops jointly is outstanding. Based on the diploms, an interesting image emerges concerning the ethnic identification of soldiers, the practice of supplementing the troops and local recruitment. The author refers to the mobility of soldiers as well: occasionally, certain people were transferred to far-away territories to complete tasks.

The author makes a note concerning the practice of certain constitutions to enlist units in numerical rank, bypassing topographic ot criptotopographic orders.
The author describes 85 military establishments altogether; among them, there were two legionary and 44 auxiliary forts, 32 smaller fortifications and numerus-sized camps, and 7 objects of unknown type.

The topic of the work is broadened with a chapter on the religion of the Raetian Roman army that, on the basis of dedications, investigates not only the cults of deities enjoying empire-wide recognition and of local gods, but also deals with data regarding collective cult practices of communities (e.g. collegia veteranorum). We may be grateful for the author for making a summary of available results concerning military personalities, the financement of restorations and expansions of sanctuaries; such inscriptions are frequent in Pannonia as well.

The last chapter is an epigraphic data-based overview of the elements of the military rank hierarchy of the Roman army. Among officers of higher ranks (legati, praefecti, praepositi, tribuni), he encounters and presents in the form of a chartfour legion commanders, and 36 commanders of auxiliary troops. At high-rank officers - as he demonstrates - there were greater possibilities for mobility; for instance, one of the legio commanders served in 4 different legions. In the case of lower-rank officers, with the help of pie charts the author presents the ratio of persons known by epitaphs, votive or instrumentum inscriptions. Usually, these officers spent their service time at the same unit.

At the auxiliary corps of the province consisting of 9.5-10 thousand heads, relatively few inscriptions deal with ordinary soldiers (eques, miles); on the basis of inscriptions, we have data about 27 soldiers altogether. From data provided by other provinces, we already know that transferred units were complemented locally (cives Raeti militantes in cohorte II Tungrorum). The author pays attention to the later fate of retired soldiers as well; veterans participated in rebuilding works of santuaries in numerous cases. The coherence and cooperation of veterans is evidenced by collegium veteranorum inscriptions.

The last chapter discusses the promotions of soldiers, their participation in special units (singulares), and the process of electing the commanders of united troops. An important chapter deals with the construction works of soldiers, inscriptions reporting the building of roads, bridges and forts. These works took place mostly after 141, but most of all, in the first half of the $3^{\text {rd }}$ century. The matter of the constructions is often unknown, as many inscriptions were placed on the facades of buildings, so for contemporaries, references of the notes were obvious. The author rightly supposes that particular units fulfilled their construction duties in previously settled locations.

The publication of the book is important not only for the military history, but also for the history of events, social processes and even of religious cults of the province.

Dénes Gabler Hungarian Academy of Sciences, Research Centre for Humanities Institute for Archaeology

Úri u.49, Budapest, H-1014, Hungary Gabler.Denes@btk.mta.hu 
Jürgen Trumm-Matthias Flück: Am Südtor von Vindonissa. Gesellschaft pro Vindonissa, Brugg 2013. 399 Bilder, Abbildungen, Tafeln und Diagramme, 6 Grundrisse und Profile. 530 Seiten +566 Seiten im digitalen Teil.

Im Süden des römischen Legionslagers Vindonissa (heute: Windisch, Kanton Aargau, Schweiz) führte die Kantonsarchäologie Aargau in den Jahren 2003-2006 eine großflächige Ausgrabung durch. In der Publikation Am Südtor von Vindonissa liegen die Ergebnisse solcher Untersuchungen im Mittelpunkt, wie die Umwehrung des Legionslagers, die Steinbauten der 21. und 11. Legion und jüngere Befunde nach Auflassung der Garnison. Die Verfasser des Bandes, Jürgen Trumm und Matthias Flück, waren mit Wissenschaftlerinnen und Wissenschaftlern von verschiedensten Untersuchungsbereichen am Projekt Vindonissa beteiligt. Durch die modernsten Methoden geben sie uns einen neuen Einblick ins Leben einer Garnison im südlichen Obergermanien aus dem ersten Jahrhundert.

Nach mehr als hundert Jahren archäologischer Feldforschung im Legionslager Vindonissa gibt es nur noch wenige weiße Flächen. In den ersten Jahrzehnten des 20. Jahrhunderts war Samuel Heuberger der Pionier, der veritable Forschungsgrabungen in Vindonissa durchführte. Heuberger und andere Wissenschaftler haben mehrere, zumeist etwa $1 \mathrm{~m}$ breite und bis zu $80 \mathrm{~m}$ lange Sondierungsgräben angelegt. Diese frühen Ausgrabungen führten zur Lokalisierung des Südtors, der Straßen, Lagermauern, Kanälen und mehrerer wichtiger Orientierungspunkte. Erst 1953 wurden weitere archäologische Beobachtungen dokumentiert. R. Fellmann interpretierte die Befunde unter der heutigen Scheuergasse als Tribunenhäuser und eine $25 \mathrm{~m}$ lange Doppelbaracke. In den folgenden Jahrzehnten haben die Archäologen mehrere Grabungen im Areal durchgeführt. 2003 haben sie mit der großflächigen Ausgrabung angefangen, deren Ergebnisse in diesem Band vorgestellt werden.

Die Publikation führt uns in die Methoden und Ergebnisse des Projekts in neun großen Abschnitten ein (fünf in dem Band und vier in dem digitalen Teil). Nach Zusammenfassung und Grundlagen der Forschung (technischer Grabungsbericht, Forschungsgeschichte und Kenntnisstand) geben die Autoren in der Synthese, der archäologischhistorischen Auswertung und den Exkursen eine detaillierte Beschreibung von allen Bauperioden und deren Funden. Obwohl während der Grabungen zwischen 2003 und 2006 bloß 1,3\% der Gesamtfläche des ehemaligen Lagers freigelegt wurde, können die Experten von verschiedenen Bereichen durch die Funde (Keramik, Münzen, Kleinfunde wie Fibeln, Militaria, Toilettengeräte und medizinisches Besteck oder die inschriftlichen Zeugnisse) einen gründlichen Einblick in den Alltag der Garnison von drei Legionen gewährleisten. Auch mit den Methoden der Anthropologie, Archäozoologie und Archäobotanik wurde dieses Bild immer detaillierter.
In der Grabungsperiode 2003-2006 ist eine gut erhaltene Mannschaftsbaracke (knapp $40 \mathrm{~m}$ lang) ausgegraben worden. Die gut erhaltenen Raumausstattungen und das Fundmaterial aus dieser Baracke halfen den Archäologen bei der Klassifizierung dieses Militärgebäudes. Die Mannschaftsbaracke von Vindonissa erweist sich umso wichtiger, weil vorher nur wenige Mannschaftsbaracken vollständig und mit modernen Grabungsmethoden ausgegraben worden sind. Die Betrachtungen ermöglichen es, das Lager von Vindonissa in den Kontext römischer Militärlager der Rhein- und Donauprovinzen sowie von Britannien zu stellen.

Die rekonstruierten Gebäude auf dem Grund des ehemaligen Lagers Vindonissa benutzen diese Informationen und interpretieren sie in physischer Form. Der Legionärspfad Vindonissa bietet für die Gäste des Römer-Erlebnisparks mit modernsten Inszenierungen einen leichten Zugang.

Die neuesten Ausgrabungsbefunde und die neueste Analyse datieren die wichtigsten Perioden im Leben des Lagers. Fundmünzen und Inschriften zeigen, wie die wichtigsten Baumaßnahmen den Wechsel zwischen der 13. und der 21. Legion markierten. Der Umbau des überwiegend aus Holz und Fachwerk bestehenden Legionslagers in ein weitgehend versteinertes Lager geschah um $45 \mathrm{n}$. Chr. und wurde $47 \mathrm{n}$. Chr. abgeschlossen.

Nach Aussage der Funde vollzog sich der Wechsel zwischen älterer und jüngerer Steinbauperiode zwischen 65 und $72 \mathrm{n}$. Chr. Die letzte, die 11. Legion, die in Vindonissa stationierte, kam wahrscheinlich um $71 \mathrm{n}$. Chr. an. Die 11. Legion errichtete erst einige Zeit nach den Wirren des „Vierkaiserjahres“ 68/69 n. Chr., im Verlauf der Feldzüge 74/75 n. Chr., ein neues Legionslager bei Rottweil-Area Flaviae. Einige Gruppen der Legion blieben aber in Vindonissa und die beiden Lager funktionierten zeitgleich.

Die ehemalige Garnison Vindonissa wurde um das Jahr 101 n. Chr. systematisch aufgelassen, aber das bedeutete nicht das Ende des Lagers. Das Areal verblieb noch mehrere Jahrzehnte unter Kontrolle der Militärverwaltung. Beweise dafür sind zwei Bruchstücke eines so genannten Militärdiploms und ein auf Bronze gefertigte Abschrift einer Entlassungsurkunde - ausgestellt im November des Jahres 121 oder 122 n. Chr. für den Prätorianer Secundinus aus Augusta Taurinorum (Turin)

Die neuen historisch-archäologischen Forschungen haben R. Laur-Belarts frühere Annahme bezüglich einer Wiederbefestigung des Lagers im 3. oder 4. Jahrhundert $n$. Chr. nicht bestätigt.

Die physische Form dieser großartigen Publikation wurde mit Literaturverzeichnis, Abbildungsnachweis und Impressum geschlossen. Der digitale Teil des Projekts (eine detaillierte Auswertung, Befundkatalog, Kataloge, Tafeln und Listen) findet sich im Internet (www.ag.ch/archeologie/publikationen). 